\title{
Evaluation of Antimony Tri-Iodide Crystals for Radiation Detectors
}

\author{
Toshiyuki Onodera $\mathbb{D}^{1}{ }^{1}$ Koei Baba, ${ }^{1}$ and Keitaro Hitomi ${ }^{2}$ \\ ${ }^{1}$ Department of Electrical and Electronic Engineering, Tohoku Institute of Technology, Sendai 982-8577, Japan \\ ${ }^{2}$ Cyclotron and Radioisotope Center, Tohoku University, Sendai 980-8578, Japan
}

Correspondence should be addressed to Toshiyuki Onodera; t_onodera@tohtech.ac.jp

Received 23 May 2018; Revised 5 November 2018; Accepted 14 November 2018; Published 5 December 2018

Academic Editor: Michael I. Ojovan

Copyright (C) 2018 Toshiyuki Onodera et al. This is an open access article distributed under the Creative Commons Attribution License, which permits unrestricted use, distribution, and reproduction in any medium, provided the original work is properly cited.

\begin{abstract}
This study was carried out to examine the potential of antimony tri-iodide $\left(\mathrm{SbI}_{3}\right)$ as a material for radiation detectors that operate at room temperature. $\mathrm{SbI}_{3}$ is a compound semiconductor with an $\mathrm{AsI}_{3}$-type crystal structure, high atomic number (Sb: 51, I: 53), high density $\left(4.92 \mathrm{~g} / \mathrm{cm}^{3}\right)$, and a wide band-gap energy $(2.2 \mathrm{eV})$. In addition, crystalline $\mathrm{SbI}_{3}$ is easy to grow by conventional crystal growth techniques from melting phase because the material exhibits a low melting point $\left(171^{\circ} \mathrm{C}\right)$ and undergoes no phase transition in the range of its solid phase. In this study, $\mathrm{SbI}_{3}$ crystals were grown by the Bridgman method after synthesis of $\mathrm{SbI}_{3}$ from $99.9999 \%$ pure $\mathrm{Sb}$ and $99.999 \%$ pure $\mathrm{I}_{2}$. The grown crystals consisted of several large grains with red color and were confirmed to be singlephase crystals by X-ray diffraction analysis. $\mathrm{SbI}_{3}$ detectors with a simple planar structure were fabricated using the cleavage plates of the grown crystals, and the pulse-height spectra were recorded at room temperature using an ${ }^{241} \mathrm{Am}$ alpha-particle (5.48 MeV) source. The detector showed response to the alpha-particle radiation.
\end{abstract}

\section{Introduction}

Numerous compound semiconductors have been actively investigated for use in radiation detectors [1-9]. High detection efficiency for gamma-rays as well as low-noise operation at room temperature and high charge collection efficiency are important characteristics for radiation detectors with high spectroscopic performance. Although CdTe[10], CdZnTe [11], and $\mathrm{HgI}_{2}[12]$, have recently been considered as ideal detector materials in this application, these materials are more expensive than traditional detector materials such as $\mathrm{Si}$ and $\mathrm{Ge}$ because of the difficulty associated with their crystal growth.

Antimony tri-iodide $\left(\mathrm{SbI}_{3}\right)$ is a compound semiconductor with an $\mathrm{AsI}_{3}$-type crystal structure; it has been reported to be a potential semiconductor material for radiation detectors [1]. The characteristics of $\mathrm{SbI}_{3}$ include high atomic number (Sb: 51, I: 53), high density $\left(4.92 \mathrm{~g} / \mathrm{cm}^{3}\right)$, and wide bandgap energy $(2.2 \mathrm{eV})$. The physical properties of $\mathrm{SbI}_{3}$ suggest that it can be used in radiation detectors with high detection efficiency and low-noise operation at room temperature without any cooling. Figure 1 shows the attenuation coefficients for Ge, CdTe, and $\mathrm{SbI}_{3}$ [13]. As shown in this figure, $\mathrm{SbI}_{3}$ exhibits high gamma-ray stopping power equivalent to that of $\mathrm{CdTe}$. In addition, $\mathrm{SbI}_{3}$ melts at a low temperature $\left(171^{\circ} \mathrm{C}\right)$ and exhibits no phase transition between room temperature and its melting point [14]. Thus, a single $\mathrm{SbI}_{3}$ crystal can be grown from the molten material via the conventional crystal growth technique such as the Bridgman method or the traveling molten-zone method.

The aforementioned attractive properties of $\mathrm{SbI}_{3}$ suggest that $\mathrm{SbI}_{3}$ detectors can be fabricated at low cost compared with $\mathrm{CdTe}$ and $\mathrm{CdZnTe}$ detectors. $\mathrm{BiI}_{3}$ [3] and $\mathrm{PbI}_{2}$ [4] crystals were also layered structure crystals similar to $\mathrm{SbI}_{3}$ and have been studied as a new radiation detector material. However, gamma-ray energy resolution obtained from these layered materials was poor than CdTe and CdZnTe due to low charge transport properties.

Although $\mathrm{SbI}_{3}$ is a promising material, no previous studies have reported the performance of $\mathrm{SbI}_{3}$ detectors. The objective of this work was to evaluate the potential of $\mathrm{SbI}_{3}$ as a radiation detector. 


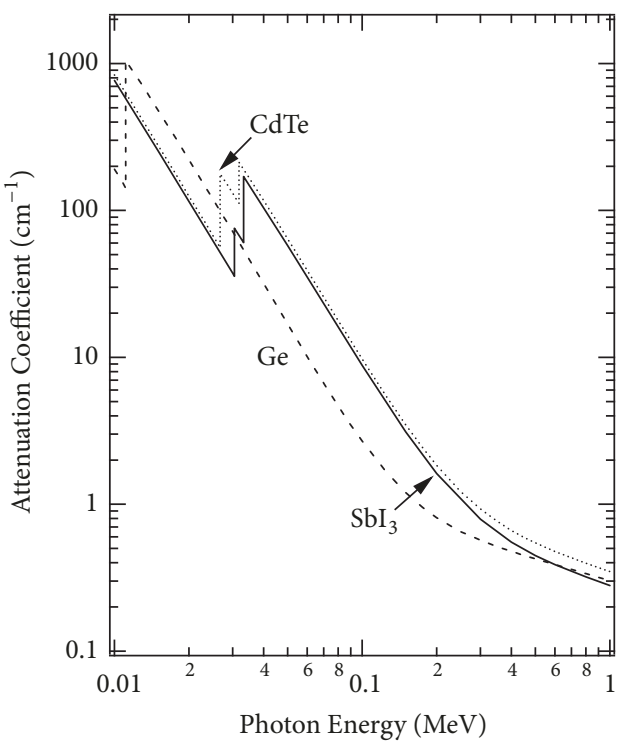

FIGURE 1: Attenuation coefficients of semiconductor detector materials as functions of the photon energy.
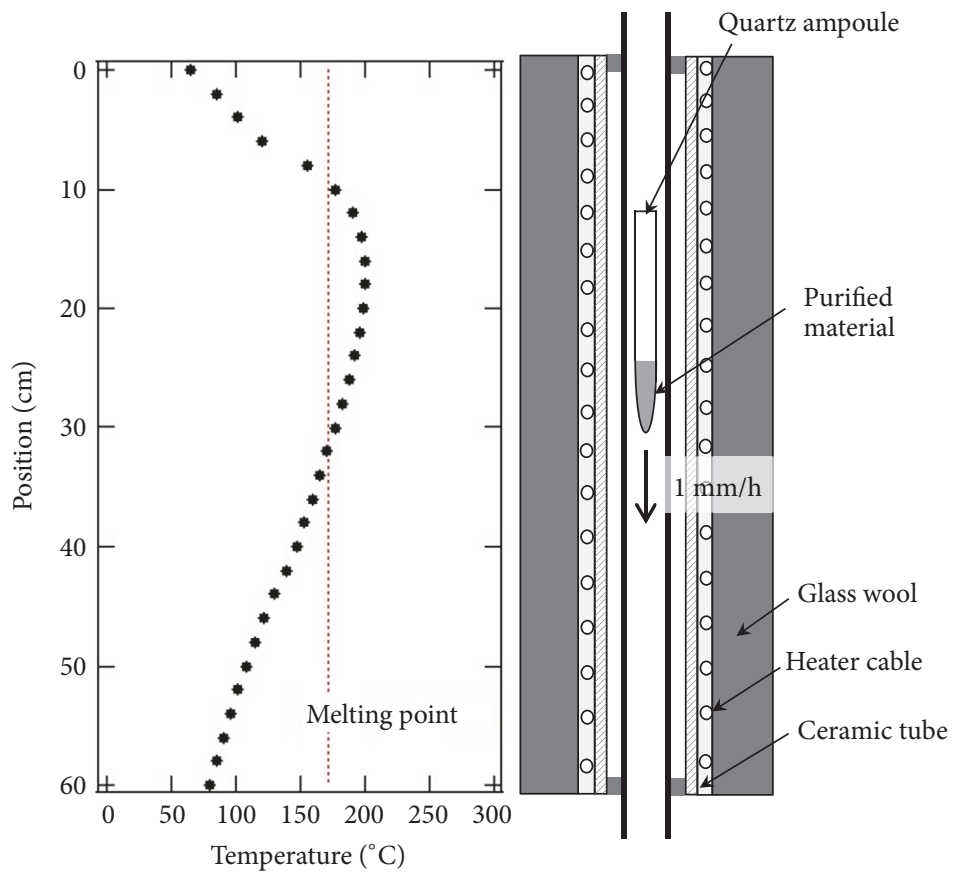

FIGURE 2: Schematic of a Bridgman furnace and the temperature distribution used in the present study.

\section{Experimental}

2.1. Crystal Growth. Commercially available Sb (nominal purity of $99.9999 \%$, Kojundo chemical laboratory Co., Ltd) and $\mathrm{I}_{2}$ (nominal purity of $99.999 \%$, Kojundo chemical laboratory Co., Ltd) were used as starting materials for growing $\mathrm{SbI}_{3}$ crystals. Stoichiometric amounts of the starting materials were loaded into a quartz ampoule $(\phi 10 \mathrm{~mm})$ that was subsequently vacuum sealed at approximately $1 \mathrm{~Pa}$. For the synthesis of $\mathrm{SbI}_{3}$, the ampoule was kept in a furnace above the melting point for $24 \mathrm{~h}$. After the synthesis, multipass zone refining was used to reduce the impurities in the synthesized material. The length of the molten zone was approximately $10 \mathrm{~mm}$. The furnace was moved at a speed of $5 \mathrm{~cm} / \mathrm{h}$, and the purification was repeated 100 times. After the material had been purified, $\mathrm{SbI}_{3}$ crystals were grown by the Bridgman method [15] at the middle section of the purified material. Figure 2 shows a schematic of the Bridgman furnace and the temperature distribution used in this study. Crystal growth was carried out in a temperature gradient of approximately $3.4^{\circ} \mathrm{C} / \mathrm{cm}$. The ampoule was located in the furnace as shown in the figure, and the material in the ampoule was completely 


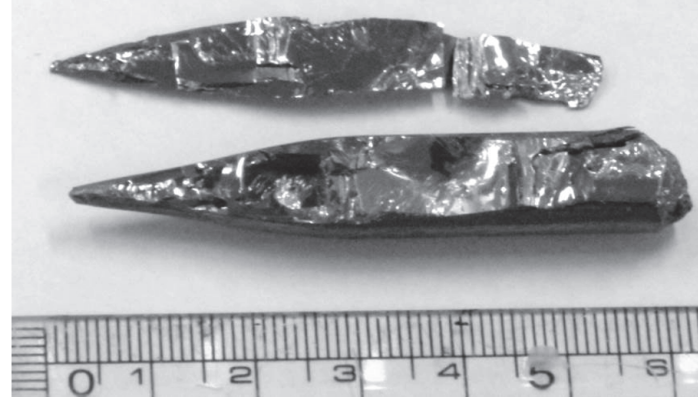

FIgURE 3: Cleaved $\mathrm{SbI}_{3}$ crystals.

melted before the ampoule was lowered. After the material had melted, the ampoule was lowered at a speed of $1 \mathrm{~mm} / \mathrm{h}$ using a stepping motor. Figure 3 shows cleaved $\mathrm{SbI}_{3}$ crystals grown in this study. The main cleavage plane was parallel to the growth direction and contained several grain boundaries. The crystal color was metallic red by reflected light and red by transmitted light, consistent with the band-gap of $\mathrm{SbI}_{3}(2.2$ $\mathrm{eV})$.

\subsection{Characterization}

2.2.1. Optical Transmittance Spectra. A UV-Vis spectrophotometer (Shimadzu UV-1800) was used to evaluate the bandgap energy of the grown $\mathrm{SbI}_{3}$ crystal. After the crystal growth, the $\mathrm{SbI}_{3}$ crystal was cleaved into thin plates (thickness of $0.1-0.3 \mathrm{~mm}$ ) using a cutter blade. Flat transparent $\mathrm{SbI}_{3}$ plates with a thickness of $0.1-0.2 \mathrm{~mm}$ were selected as samples for this evaluation.

2.2.2. X-Ray Diffraction. X-ray diffraction (XRD) analysis (Rigaku 2500HF) was used to confirm the crystallinity of the grown crystal. The voltage and current of the X-ray tube $(\mathrm{Cu}-$ $\mathrm{K} \alpha$ ) were $40 \mathrm{kV}$ and $200 \mathrm{~mA}$, respectively. Cleaved plates and powdered $\mathrm{SbI}_{3}$ were prepared from the grown crystal. Commercially available $\mathrm{SbI}_{3}$ powder was used to obtain reference data for the $\mathrm{SbI}_{3}$ phase.

2.2.3. Current-Voltage Characteristics. The current-voltage characteristics of the $\mathrm{SbI}_{3}$ detectors were measured to evaluate the resistivity of the $\mathrm{SbI}_{3}$ detectors. The bias voltage was changed from $0 \mathrm{~V}$ to $200 \mathrm{~V}$, and the leakage current of the detectors was measured at room temperature.

2.2.4. Fabrication of an $\mathrm{SbI}_{3}$ Detector. The grown crystal was cleaved into thin plates (thickness of $0.1-0.3 \mathrm{~mm}$ ), and gold electrodes (3 $\mathrm{mm}$ in diameter) were deposited onto both sides of the $\mathrm{SbI}_{3}$ crystals by the vacuum evaporation method. After electrode deposition, the $\mathrm{SbI}_{3}$ crystals were placed on a stage used for detector fabrication and palladium wires were connected to each electrode from a terminal on the stage. The detector was enclosed in an aluminum case, and the signal from the detector was taken from a BNC terminal on the case.

2.2.5. Radiation Response. For initial operation before evaluating gamma-ray detector performance of the $\mathrm{SbI}_{3}$ detectors,

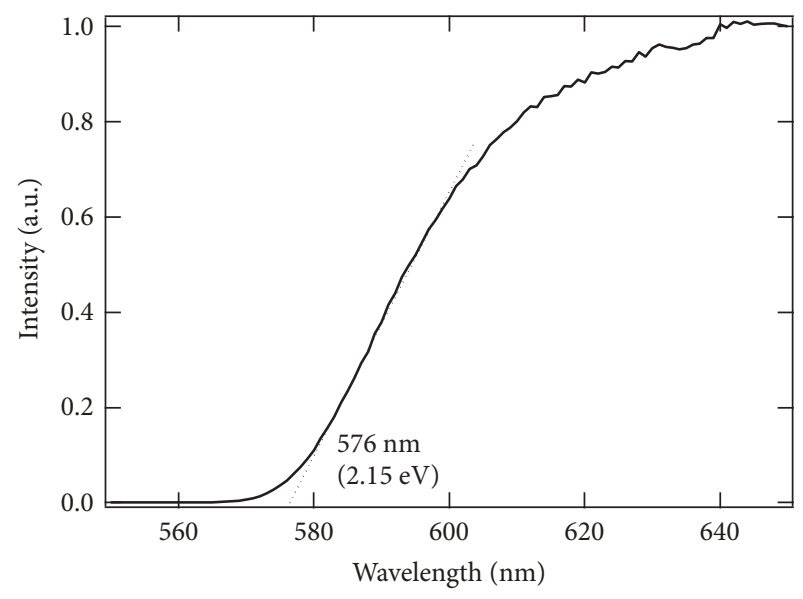

FIGURE 4: Transmittance spectrum of a cleaved $\mathrm{SbI}_{3}$ crystal.

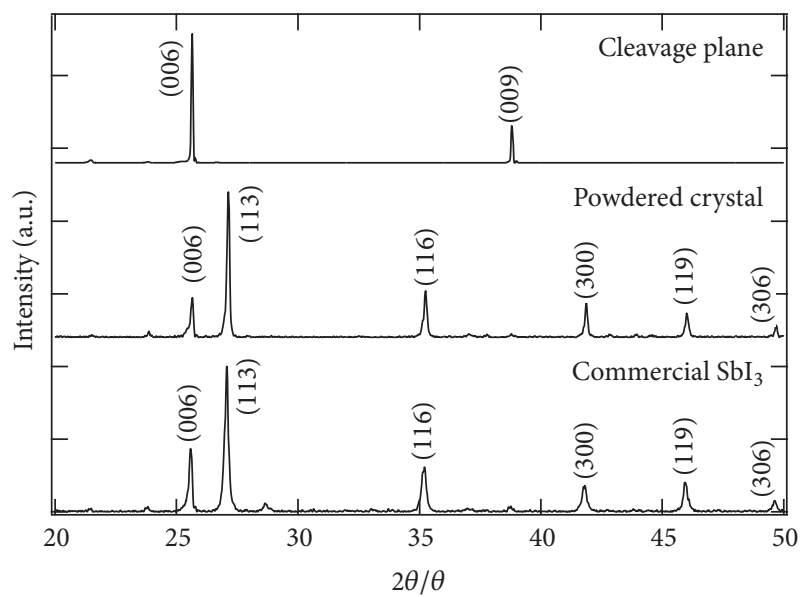

FIGURE 5: X-ray diffraction patterns of the grown $\mathrm{SbI}_{3}$ crystal and a commercially available $\mathrm{SbI}_{3}$ powder.

${ }^{241}$ Am alpha-particle energy spectra were recorded using the $\mathrm{SbI}_{3}$ detectors in a conventional measurement system comprising a preamplifier, bias supply, shaping amplifier, and a multichannel pulse-height analyzer. The ${ }^{241} \mathrm{Am}$ alphaparticle source $(185 \mathrm{kBq})$ and the detector were enclosed in the aluminum case used for the current-voltage measurements. Distance between the alpha-particle source and the $\mathrm{SbI}_{3}$ crystal was approximately $2 \mathrm{~mm}$ and the measurement was carried out in the atmosphere. After the initial evaluation, gamma-ray response was also evaluated using the same measurement system and ${ }^{137} \mathrm{Cs}$ gamma-ray source (3.7 MBq).

\section{Results and Discussion}

3.1. Characterization. Figure 4 shows the transmittance spectrum as a function of wavelength. The cutoff wavelength was approximately $576 \mathrm{~nm}$, suggesting that the band-gap energy of the grown crystal was approximately $2.15 \mathrm{eV}$, consistent with a previously reported value [1].

Figure 5 shows the XRD patterns obtained from an $\mathrm{SbI}_{3}$ plate, the powder, and the commercial $\mathrm{SbI}_{3}$ powder. All of the 


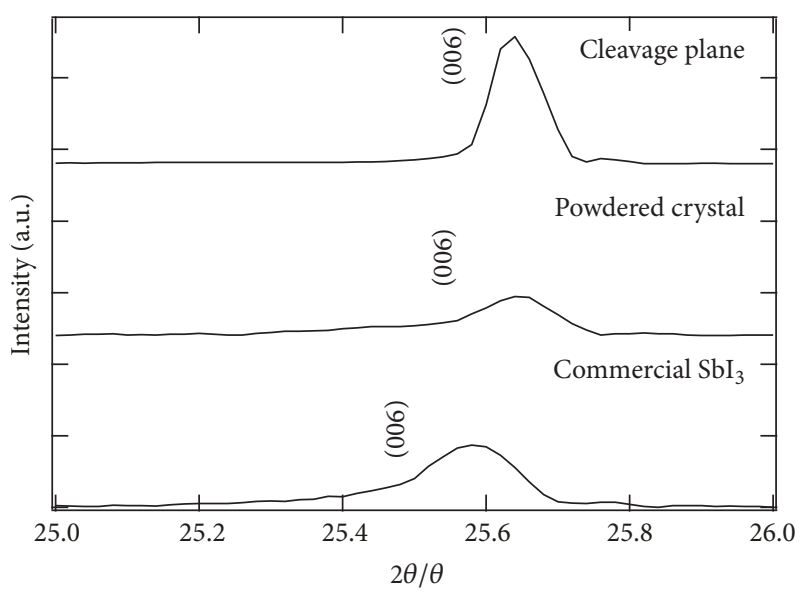

Figure 6: Magnification of the X-ray diffraction patterns in the region of the (006) reflection of $\mathrm{SbI}_{3}$.

diffraction peaks in the pattern of the powdered crystal match the JCPDS data [16], indicating that $\mathrm{SbI}_{3}$ was successfully synthesized using the starting materials and that singlecrystalline $\mathrm{SbI}_{3}$ was grown by the Bridgman method.

Figure 6 shows the extended XRD patterns in the region of the (006) plane. The peaks of the (006) plane in the pattern of the grown crystal and the powdered crystal were slightly shifted toward higher angles compared with the corresponding peak in the pattern of the commercial powder. This result implies that the lattice constant of the grown and powdered crystal samples was somewhat smaller than that of the commercial $\mathrm{SbI}_{3}$ powder. The origin of this small difference requires further investigation beyond the scope of the present work.

3.2. Detector Performance. Figure 7 shows the leakage current as a function of the bias voltage obtained from the $\mathrm{SbI}_{3}$ detectors. The thickness of the $\mathrm{SbI}_{3}$ crystal was 0.339 $\mathrm{mm}$. The resistivity of the $\mathrm{SbI}_{3}$ detectors was estimated to be approximately $1.0 \times 10^{10} \Omega \cdot \mathrm{cm}$.

Figures 8 and 9 show the alpha-particle energy spectra obtained from a typical $\mathrm{SbI}_{3}$ detector at room temperature. The thickness of the detector was $0.229 \mathrm{~mm}$. The spectra were obtained by changing the polarity of the bias voltage to evaluate the difference in charge transport properties between electrons and holes. The bias voltage was $200 \mathrm{~V}$, and the amplifier shaping time was $30 \mu \mathrm{s}$. Both energy spectra recorded under alpha-particle irradiation show increased counts above the noise counts. Penetration depth of $5.48 \mathrm{MeV}$ alpha-particles is calculated to be less than $40 \mu \mathrm{m}$ in water and the range for the alpha-particles is much smaller than the crystal thickness. Because the penetration depth of alphaparticles into the crystals was very shallow, the maximum count channel in the energy spectra depends on the transport properties of holes or electrons. As shown in Figures 8 and 9, the maximum count channel was independent of the polarity of the bias voltage. Thus, the charge transport properties of electrons and holes in the $\mathrm{SbI}_{3}$ detector were approximately equivalent. In addition, because the $\mathrm{SbI}_{3}$ detector exhibited

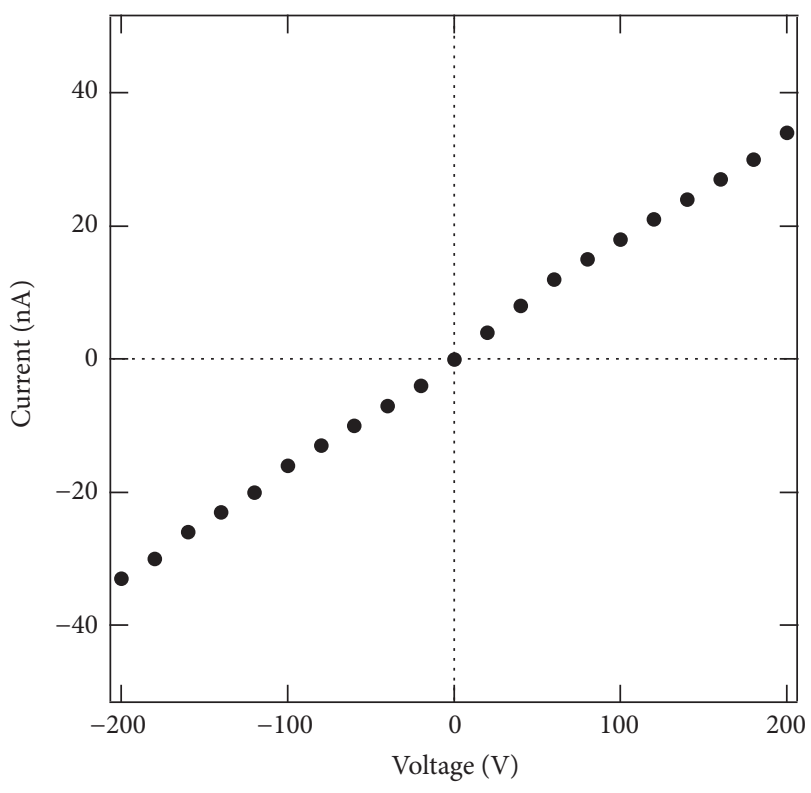

Figure 7: Current-voltage characteristics of an $\mathrm{SbI}_{3}$ detector at room temperature.

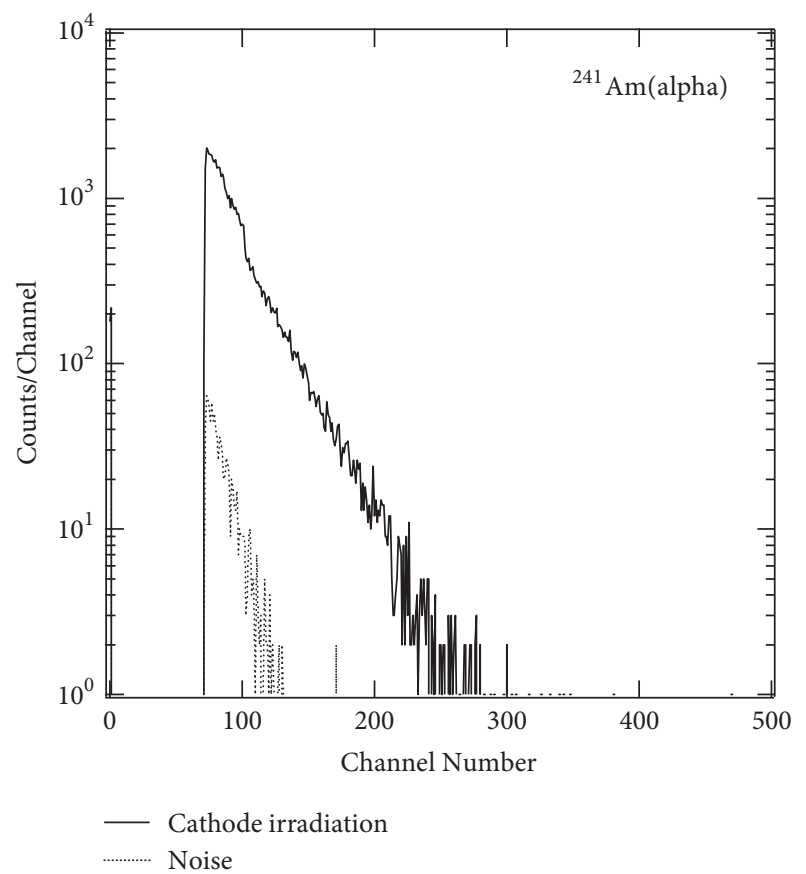

FIGURE 8: Alpha-particle energy spectrum of the $\mathrm{SbI}_{3}$ detector under cathode irradiation (electrons mainly transport in the crystal).

no full-energy peaks corresponding to $5.48 \mathrm{MeV}$ of alphaparticles, the $\mu \tau$ products for both carriers in the $\mathrm{SbI}_{3}$ detector were substantially smaller than those for other compound semiconductor detectors such as CdTe, CdZnTe, and TlBr.

Figure 10 was the alpha-particle energy spectra acquired for $30 \mathrm{~s}$ and $180 \mathrm{~s}$ and total counts were 15,834 counts and 50,969 counts for $30 \mathrm{~s}$ and $180 \mathrm{~s}$, respectively. Although the count rate linearity could not be observed from the $\mathrm{SbI}_{3}$ 


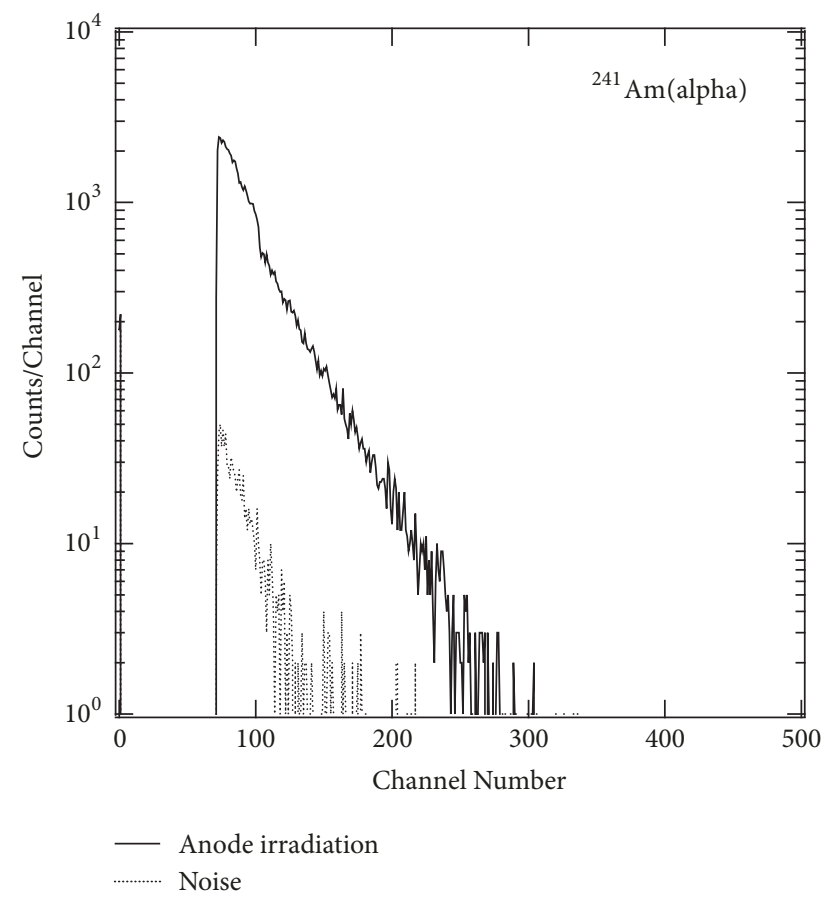

FIGURE 9: Alpha-particle energy spectrum obtained from the $\mathrm{SbI}_{3}$ detector under anode irradiation (holes mainly transport in the crystal).

detector, the linearity may be improved by reducing charge trapping and detector noise during operation.

Figure 11 shows the ${ }^{137} \mathrm{Cs}$ gamma-ray energy spectra obtained from the $\mathrm{SbI}_{3}$ detector at room temperature. Counts from the detectors were slightly increased by the irradiation of gamma-rays. These results suggested that almost gammarays from the source were penetrating through the detector because the thickness of the $\mathrm{SbI}_{3}$ crystal was $0.229 \mathrm{~mm}$.

3.3. Detector Stability. Stable detector performance is an important characteristic for radiation detectors. During operation of semiconductor detectors, temporal changes in detector performance known as the polarization phenomenon are usually observed as a decrease in pulse height. This phenomenon depends on the semiconductor material used for the detector and has been previously observed for CdTe detectors and $\mathrm{TlBr}$ detectors. Methods for improving or suppressing this phenomenon have been reported [17-20]. As previously mentioned, confirmation of the polarization phenomenon and research overcoming the phenomenon are necessary for realizing semiconductor detectors suitable for applications such as medical devices and industrial imaging.

In the present study, the $\mathrm{SbI}_{3}$ detector was continuously operated at room temperature and alpha-particle energy spectra were recorded as a function of time to evaluate the polarization phenomenon in the detector. Figure 12 shows the operation-time dependence of the ${ }^{241} \mathrm{Am}$ alpha-particle energy spectra obtained using the $\mathrm{SbI}_{3}$ detector. The spectral response of the $\mathrm{SbI}_{3}$ detector was stable for almost $24 \mathrm{~h}$ under the bias voltage $(200 \mathrm{~V})$. Although a reduction in pulse height was observed after $90 \mathrm{~h}$ of operation, the pulse

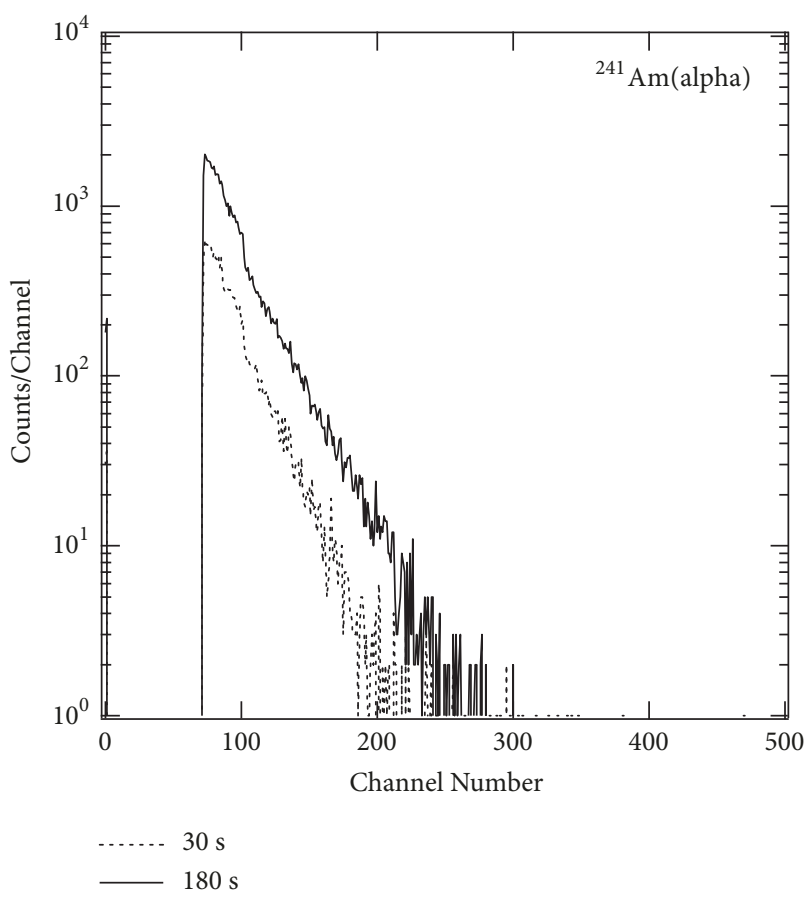

FIGURE 10: Alpha-particle energy spectrum obtained from the $\mathrm{SbI}_{3}$ detector under cathode irradiation for $30 \mathrm{~s}$ and $180 \mathrm{~s}$.

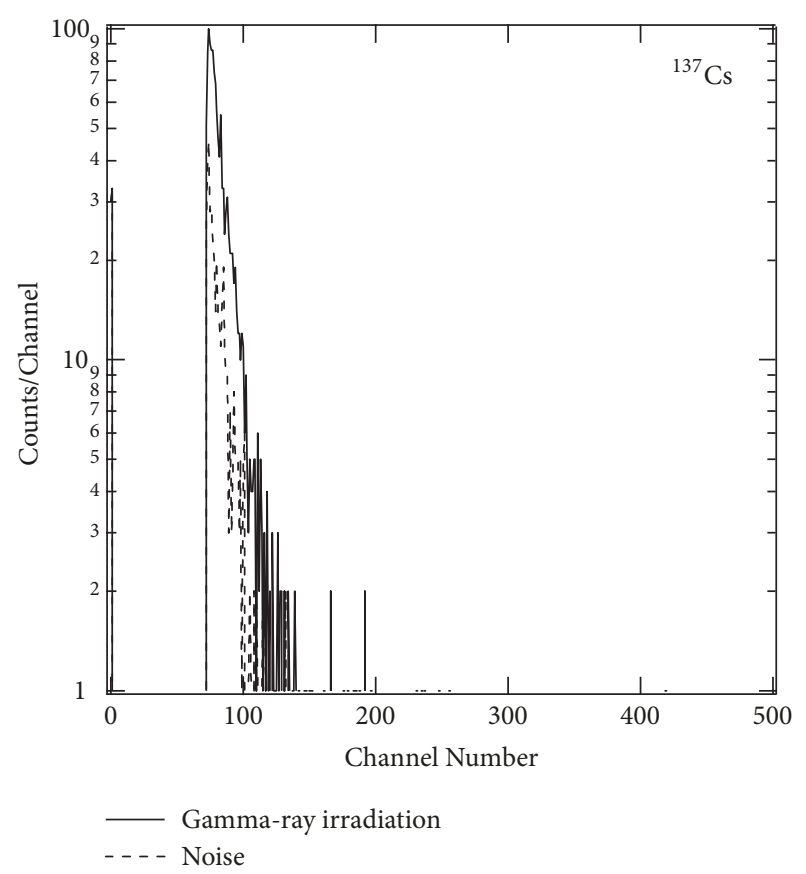

FIGURE 11: ${ }^{137}$ Cs gamma-ray energy spectrum obtained from the $\mathrm{SbI}_{3}$ detector.

height immediately recovered when the bias voltage was cutoff. Recovery from the polarization phenomenon after cutoff of the applied bias voltage has also been reported for $\mathrm{CdTe}$ detectors [10], whose recovery behavior differs from that of $\mathrm{TlBr}$ detectors. $\mathrm{TlBr}$ detectors required a long time to recover their performance because the accumulation of ionic 


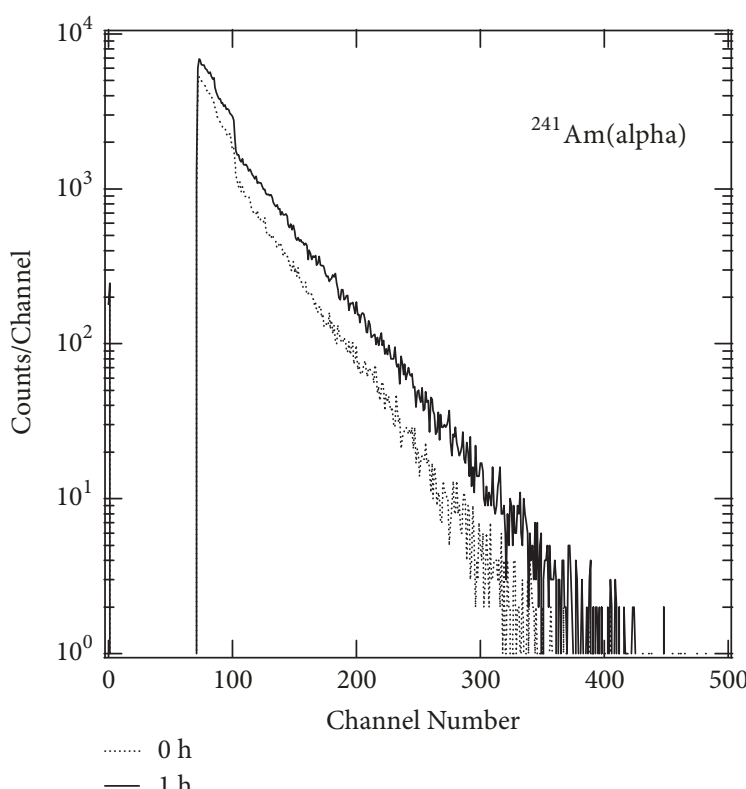

(a)

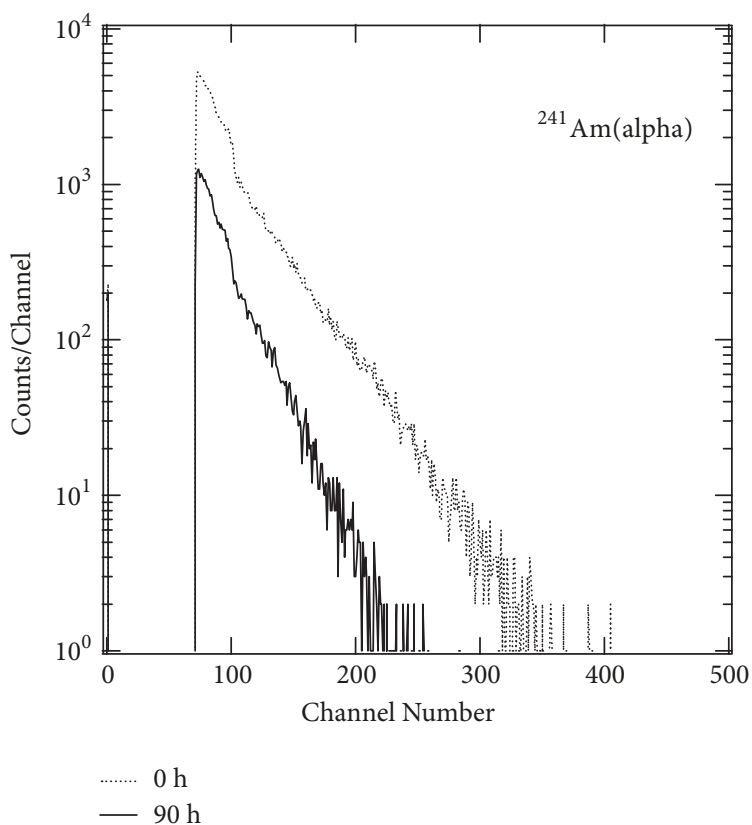

(c)

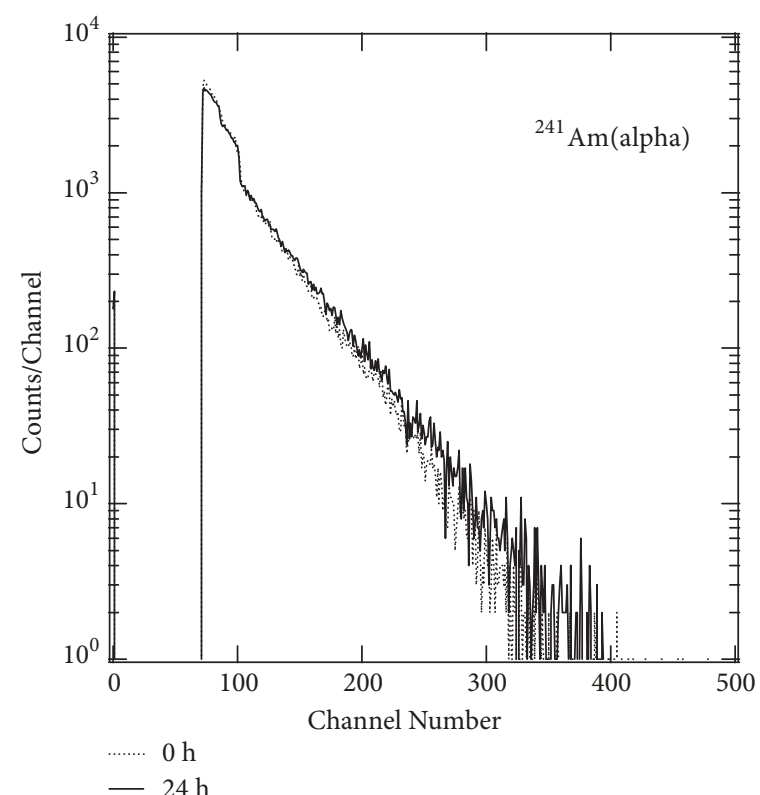

(b)

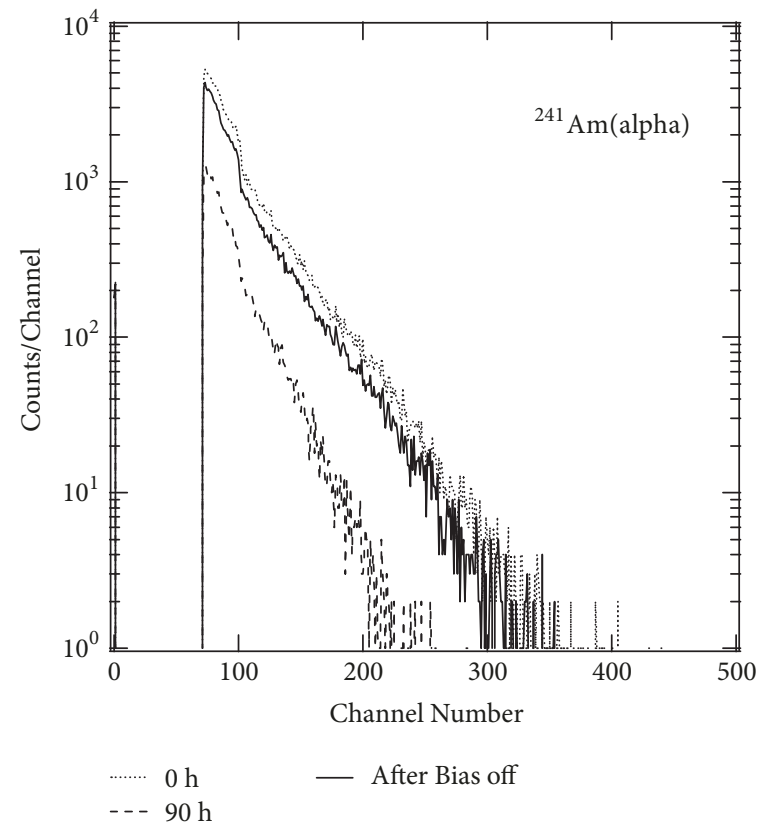

(d)

FIGURE 12: Alpha-particle energy spectra obtained from the $\mathrm{SbI}_{3}$ detector as a function of operation time at room temperature.

charge at the electrodes caused the polarization phenomenon. Therefore, the polarization phenomenon observed for the $\mathrm{SbI}_{3}$ detector is similar to that of CdTe detectors and is speculatively attributed to trapped charges.

\section{Conclusion}

$\mathrm{SbI}_{3}$ crystals were grown by the Bridgman method, and the performance of $\mathrm{SbI}_{3}$ detectors was evaluated on the basis of alpha-particle energy spectra recorded at room temperature. Although the spectra recorded using the fabricated $\mathrm{SbI}_{3}$ detectors showed no full-energy peak of the alpha-particles, the detector responded to alpha-particle irradiation. The $\mathrm{SbI}_{3}$ detector exhibited a decrease in pulse height during longterm operation, similar to other semiconductor detectors based on CdTe and TlBr; however, stable operation for 24 $\mathrm{h}$ was observed and the pulse height immediately recovered when the applied bias was cut off. Thus, $\mathrm{SbI}_{3}$ is a promising material for use in radiation detectors that exhibit stable operation. Further research on purification of the starting material and on improving carrier transport is required to improve the spectroscopic performance of the $\mathrm{SbI}_{3}$ detectors. 


\section{Data Availability}

The data used to support the findings of this study are available from the corresponding author upon request.

\section{Conflicts of Interest}

The authors declare that they have no conflicts of interest.

\section{References}

[1] G. A. Armantrout, S. P. Swierkowski, J. W. Sherohman, and J. H. Yee, "What Can Be Expected from High-Z Semiconductor Detectors?" IEEE Transactions on Nuclear Science, vol. 24, no. 1, pp. 121-125, 1977.

[2] T. Onoclera, K. Hitomi, and T. Shoji, “Temperature dependence of spectroscopic performance of thallium bromide X- and gamma-ray detectors," IEEE Transactions on Nuclear Science, vol. 54, no. 4, pp. 860-863, 2007.

[3] M. Matsumoto, K. Hitomi, T. Shoji, and Y. Hiratate, "Bismuth tri-iodide crystal for nuclear radiation detectors," IEEE Transactions on Nuclear Science, vol. 49, no. 5, pp. 2517-2520, 2002.

[4] V. Deich and M. Roth, "Improved performance lead iodide nuclear radiation detectors," Nuclear Instruments and Methods in Physics Research Section A: Accelerators, Spectrometers, Detectors and Associated Equipment, vol. 380, no. 1-2, pp. 169-172, 1996.

[5] P. Sfffert, A. Cornet, R. Stuck, R. Triboulet, and Y. Marfaing, "Cadmium telluride nuclear radiation detectors," IEEE Transactions on Nuclear Science, vol. 22, no. 1, pp. 211-225, 1975.

[6] M. Schieber, T. E. Schlesinger, R. B. James, and H. Hermon, "Study of impurity segregation, crystallinity, and detector performance of melt-grown cadmium zinc telluride crystals," Journal of Crystal Growth, vol. 237-239, no. 1, pp. 2082-2090, 2002.

[7] M. Schieber, "Fabrication of $\mathrm{HgI}_{2}$ nuclear detectors," Nuclear Instruments and Methods in Physics Research Section B: Beam Interactions with Materials and Atoms, vol. 144, no. 3, pp. 469477, 1977.

[8] M. R. Squillante, C. Zhou, J. Zhang, L. P. Moy, and K. S. Shah, "InI Nuclear Radiation Detectors," IEEE Transactions on Nuclear Science, vol. 40, no. 4, pp. 364-366, 1993.

[9] M. Kocsis, "Proposal for a new room temperature x-ray detector-thallium lead iodide," IEEE Transactions on Nuclear Science, vol. 47, no. 6 I, pp. 1945-1947, 2000.

[10] P. Siffert, J. Berger, C. Scharager et al., "Polarization in cadmium telluride nuclear radiation detectors," IEEE Transactions on Nuclear Science, vol. 23, no. 1, pp. 159-170, 1976.

[11] T. E. Schlesinger, J. E. Toney, H. Yoon et al., "Cadmium zinc telluride and its use as a nuclear radiation detector material," Materials Science and Engineering: R: Reports, vol. 32, no. 4-5, pp. 103-189, 2001.

[12] H. A. Lamonds, "Review of mercuric iodide development program in Santa Barbara," Nuclear Instruments and Methods in Physics Research Section B: Beam Interactions with Materials and Atoms, vol. 213, no. 1, pp. 5-12, 1983.

[13] M. J. Berger and J. H. Hubbell, "XCOM: Photon cross sections on a personal computer," Center for Radiation Research, National Bureau of Standards NBSIR-87-3597, Gaithersburg, MD, 1987.
[14] F. M. Jaeger and H. J. Doornbosch, "Die jodide der elemente aus der stichstoffgruppe," Z. anorg. u. allgem. Chem., vol. 75, no. 1, pp. 261-271, 1912.

[15] P. W. Bridgman, "Certain Physical Properties of Single Crystals of Tungsten, Antimony, Bismuth, Tellurium, Cadmium, Zinc, and Tin," Proceedings of the American Academy of Arts and Sciences, vol. 60, no. 6, pp. 305-383, 1925.

[16] “Powder diffraction file (No. 70273)," JCPDS, 1997.

[17] H. L. Malm and M. Martini, "Polarization phenomena in CdTe nuclear radiation detectors," IEEE Transactions on Nuclear Science, vol. 21, no. 1, pp. 322-330, 1974.

[18] D. Vartsky, M. Goldberg, Y. Eisen et al., "Radiation induced polarization in CdTe detectors," Nuclear Instruments and Methods in Physics Research Section A: Accelerators, Spectrometers, Detectors and Associated Equipment, vol. 263, no. 2-3, pp. 457462, 1988.

[19] T. Onodera, K. Hitomi, and T. Shoji, "Spectroscopic performance and long-term stability of thallium bromide radiation detectors," Nuclear Instruments and Methods in Physics Research Section A: Accelerators, Spectrometers, Detectors and Associated Equipment, vol. 568, no. 1, pp. 433-436, 2006.

[20] K. Hitomi, T. Shoji, and Y. Nizeki, "A method for suppressing polarizarion phenomena in $\mathrm{TlBr}$ detectors," Nuclear Instruments and Methods in Physics Research Section A: Accelerators, Spectrometers, Detectors and Associated Equipment, A585, pp. 102-104, 2008 

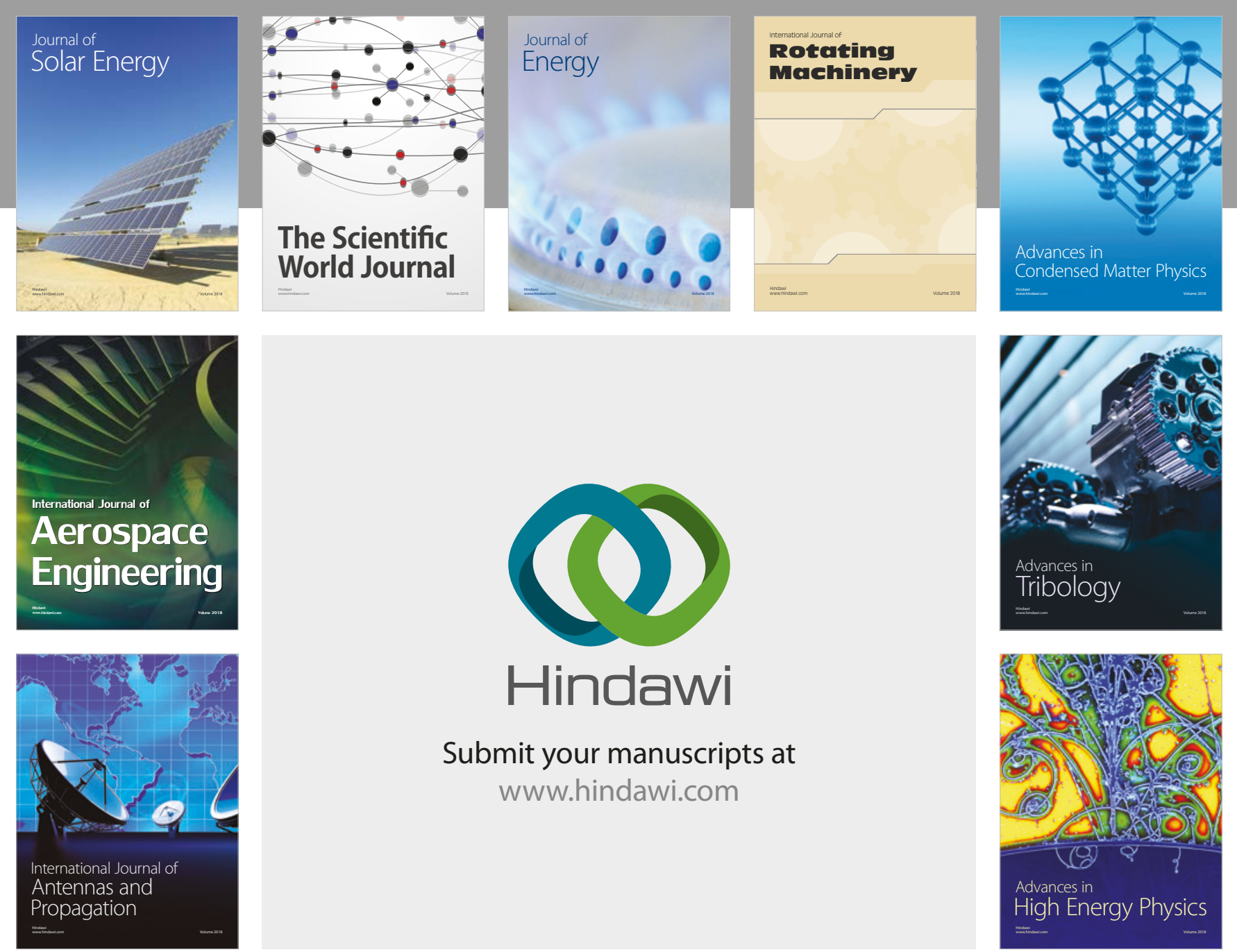

Submit your manuscripts at

www.hindawi.com
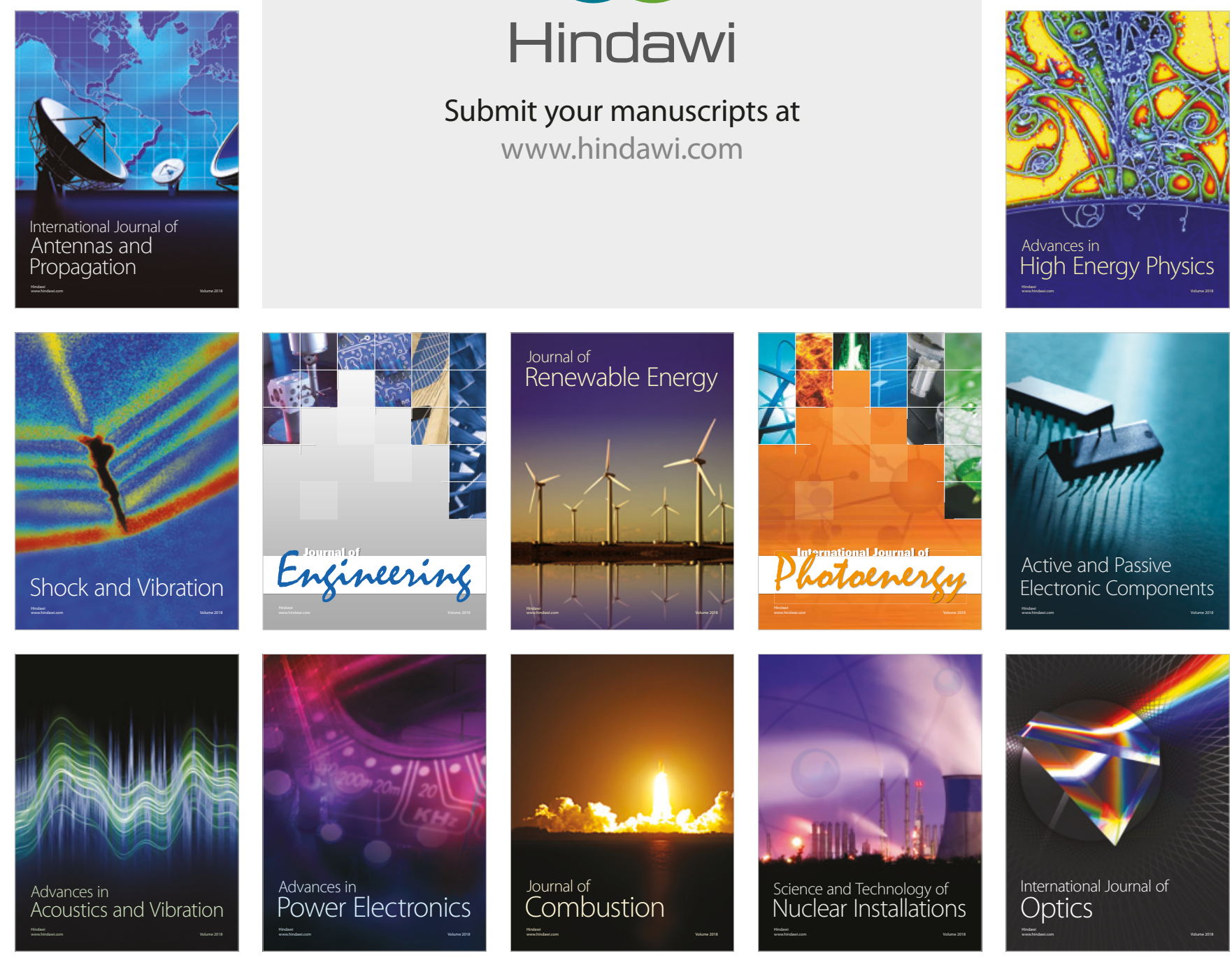